\title{
LA POBLACIÓN VALENCIANA DURANTE LA DÉCADA 1981-1991
}

\author{
Vicente Gozálvez Pérez
}

\author{
RESUMEN
}

La evolución del censo de la población valenciana durante los años 1980 ofrece un balance relativamente positivo, debido al incremento reciente de la inmigración neta. En el movimiento natural, la natalidad y fecundidad continúan el descenso iniciado en 1977, mientras la mortalidad, baja por el rejuvenecimiento demográfico producido por la masiva inmigración recibida entre 1960 y 1975, comienza a aumentar durante el último quinquenio debido al envejecimiento progresivo; como resultado, el excedente natural positivo se ve seriamente comprometido. Se acentúa la concentración demográfica en los municipios urbanos, mientras recientemente se intensifica la despoblación de las áreas rurales más desfavorecidas.

\section{RÉSUMÉ}

L'évolution du recensement de la population valencienne pendant les années 1980 offre un bilan relativement positif, dû à la récente poussée de l'inmigration. Dans le mouvement naturel, la natalité et la fécondité poursuivent leur chute depuis 1977, tandis que la mortalité, faible à cause du rajeunissement démographique provoqué par la massive inmigration des années 1960 à 1975, commence à augmenter récenmment, dû au vieillissement; le resultat est la mise en danger de l'excedent naturel positif. La concentration démographique s'accentue dans les communes urbaines, tandis que la depopulation s'intensifie dans les zones rurales les plus défavorisées.

En 1991 el País Valenciano censa 3.923.841 habitantes de hecho, que suponen una ganancia de 277.076 sobre el censo de $1981(+7,6 \%)$. Aunque dicho incremento apenas si supera la mitad del registrado en cada una de las dos décadas anteriores (573.594 habitantes en 1970-81; 592.292 en 1960-70), debemos darlo por saneado en el contexto de las regiones españolas: sólo Andalucía y Madrid consiguen crecimientos absolutos mayores que el del País Valenciano, mientras en el extremo opuesto Asturias, Castilla y León, Galicia y el País Vasco han disminuido sus censos demográficos entre 1981 y 1991; sobre el censo total nacional de 1991, la Comunidad Valenciana participa con el 9,9\%, mientras en las ganancias demográficas totales de la década, la Comunidad participa con el 16,4\%. Especial atención merece la evolución censal durante el último quinquenio: en 1986-91 


\section{CUADRO I}

PAÍS VALENCIANO. EXCEDENTE NATURAL Y MIGRACIONES NETAS, 1981-1991

\begin{tabular}{|c|c|c|c|c|c|c|}
\hline Período & $\begin{array}{l}\text { Población hecho } \\
\text { primer año }\end{array}$ & $\begin{array}{c}\text { Excedente } \\
\text { natural }\end{array}$ & $\begin{array}{l}\text { Población hecho } \\
\text { último año }\end{array}$ & $\begin{array}{c}\text { Saldo } \\
\text { migratorio }\end{array}$ & $\begin{array}{c}\text { Crecimiento } \\
\text { censal } \\
\text { habitantes }\end{array}$ & $\%$ \\
\hline \multicolumn{7}{|c|}{ Provincia de Alicante } \\
\hline $1981-86$ & 1.148 .597 & 38.573 & 1.254 .920 & 67.750 & 106.323 & 9,3 \\
\hline 1986-91 & 1.254 .920 & 23.819 & 1.334 .545 & 55.806 & 79.625 & 6,3 \\
\hline \multicolumn{7}{|c|}{ Provincia de Castellón } \\
\hline $1981-86$ & 431.755 & 6.125 & 437.320 & -560 & 5.565 & 1,3 \\
\hline $1986-91$ & 437.320 & 379 & 448.182 & 10.483 & 10.862 & 2,5 \\
\hline \multicolumn{7}{|c|}{ Provincia de Valencia } \\
\hline $1981-86$ & 2.066 .413 & 46.110 & 2.079 .762 & -32.761 & 13.349 & 0,6 \\
\hline 1986-91 & 2.079 .762 & 16.025 & 2.141 .114 & 45.327 & 61.352 & 2,9 \\
\hline \multicolumn{7}{|c|}{ País Valenciano } \\
\hline $1981-86$ & 3.646 .765 & 90.808 & 3.772 .002 & 34.429 & 125.237 & 3,4 \\
\hline 1986-91 & 3.772 .002 & 40.223 & 3.923 .841 & 111.616 & 151.839 & 4,0 \\
\hline
\end{tabular}

FuENTE: Censos de población; Movimiento natural de la población.

Los datos de movimiento natural de 1989 y 1990 son provisionales.

tanto la nación como doce comunidades autónomas han evolucionado con tendencia negativa respecto a 1981-86, es decir han crecido menos o han aumentado las pérdidas censales; sólo Aragón, Cataluña, Comunidad Valenciana, Madrid y Navarra han tenido un incremento censal mayor en el último quinquenio.

El comportamiento de los componentes del crecimiento demográfico merece ser subrayado: el excedente natural (nacimientos menos defunciones) continúa acentuando su deterioro; si durante el quinquenio 1981-85 fue de 90.808 habitantes para el País Valenciano, en 1986-90 se ha reducido a 40.223. A efectos comparativos, en 1976-80 el excedente natural de la región fue de 163.539 habitantes. La reducida cifra del quinquenio 1986-90 se debe fundamentalmente a la disminución de nacimientos, pero también al incremento de las defunciones a resultas del considerable aumento de la población vieja propiciada por la evolución de la pirámide de edades; el incremento de los óbitos en 1986-90 es una novedad respecto a la década 1976-85, cuyos dos quinquenios registran cifras idénticas.

El saldo migratorio es el segundo componente del crecimiento censal; en el conjunto del País Valenciano la inmigración neta suma algo más de la mitad - 53\% - del incremento demográfico durante la década, aunque con valores muy distintos en cada uno de los dos quinquenios, pues el saldo migratorio fue de +34.429 habitantes en 1981-85 y de +111.616 en 1986-90 (Cuadro I), lo que supone una reactivación inmigratoria, aunque en modo alguno podamos concluir que se trata de una inmigración atraída por el incremento de la oferta de empleo, como sucedió en 1960-75, a juzgar por los cambios en la distribución por edades de la población entre 1981 y 1991; de todos modos sería preciso realizar estudios sobre las características demográficas y sociales de esta inmigración reactivada. 


\section{Desigualdad en el reparto espacial del incremento demográfico}

Las tres provincias valencianas muestran durante la década 1981-91 comportamientos bien distintos tanto en sus crecimientos censales como en la evolución de los factores que los propician. Como dato previo, hay que tener presente la participación relativa que tiene cada provincia en el censo regional de 1991: Valencia alcanza el 54,6\%, Alicante el 34,0 y Castellón el 11,4\%. Durante la década, Alicante aumentó su censo un 16,2\%, Castellón $3,8 \%$ y Valencia 3,6\%, aunque estas cifras decenales son, a su vez, bien distintas en cada uno de los dos quinquenios.

Alicante ha concentrado la mayor parte del crecimiento demográfico del País Valenciano, el 67\% del total de la década, aunque durante 1981-86 llegó a totalizar el 85\% regional. Las tendencias provinciales han cambiado durante el último quinquenio, pues aunque Alicante continúe registrando más de la mitad de todo el incremento regional, su peso relativo ha disminuido, mientras Castellón y Valencia han mejorado sustancialmente sus desastrosos balances de 1981-86 (vid. Cuadro I), debido exclusivamente a la inmigración neta.

El excedente natural se ha reducido en las tres provincias bien que con intensidad muy desigual: así, entre 1981-85 y 1986-90 Alicante lo disminuyó un 38\%, Valencia un 65\% y Castellón un $94 \%$, pues en esta última circunscripción estas cifras han sido 6.125 y 379 habitantes en cada uno de los dos quinquenios. Los saldos migratorios han tenido, en cambio, un comportamiento inverso; en el período 1981-85 el saldo migratorio aún fue muy saneado en Alicante (+67.750), mientras era negativo en Castellón y sobre todo en Valencia (-32.761). En 1986-90 Alicante continúa con el mayor saldo inmigratorio (+55.806), pero ahora es sólo la mitad del total regional y con clara tendencia a su disminución; en contraposición el centro y norte del País han cambiado sus saldos migratorios negativos por positivos. Así la inmigración neta, durante el último quinquenio, se ha erigido en las tres provincias en el protagonista del crecimiento censal pues representa más del 70\%, y en Castellón, además, es el único factor de su aumento demográfico.

\section{El movimiento natural: se acentúa la reducción del excedente vegetativo}

\subsection{Natalidad y fecundidad}

El País Valenciano es la región que durante la década de los años 1980 tiene tasas más próximas a la media nacional, aunque las diferencias entre el norte y sur del País son considerables y se corresponden a escala local con las que se dan entre el norte y sur de España, bien que éstas son, lógicamente, mucho más acentuadas ${ }^{1}$. Así, mientras el número de nacimientos del País Valenciano se ha reducido en un 27\% entre 1981 y 1990, en Castellón esta cifra fue del 34\%, en Valencia 28\% y en Alicante 23\%; como resultado, la natalidad de 1990 es de 8,8 por 1.000 en Castellón, 10,1 en Valencia y 11,2 en Alicante, mientras la media nacional es de 10,2, tasa que, como es sabido, se sitúa por debajo de todos los países desarrollados — p.e. en 1990 la natalidad es en Francia de 13,4 por 1.000, en Reino Unido 13,9, en Suecia 14,5² —, con la excepción de Japón, Grecia e Italia (9,8 por

1 GOZÁlVEZ PÉREZ, V.: «Crise et contrastes spatiaux de la fécondité espagnole», Espace. Populations. Societés, 1989, núm. 2, pp. 201-214.

2 GUIBERT-LANTOINE, C. et MONNIER, A.: «La conjoncture démographique: l'Europe et les pays développés d'Outre-Mer», Population, 4, 1992, pp. 1.017-1.036. 
CuAdro II

PAÍS VALENCIANO. TASAS DE FECUNDIDAD POR EDADES, EN \%;; AÑNS 1975 Y 1986

\begin{tabular}{|c|c|c|}
\hline Edad de la madre & $\begin{array}{l}\text { Tasa } \\
1975\end{array}$ & 1986 \\
\hline 15 & 2 & 2 \\
\hline 16 & 7 & 6 \\
\hline 17 & 13 & 12 \\
\hline 18 & 26 & 19 \\
\hline 19 & 38 & 29 \\
\hline 20 & 60 & 35 \\
\hline 21 & 90 & 46 \\
\hline 22 & 137 & 62 \\
\hline 23 & 180 & 81 \\
\hline 24 & 221 & 103 \\
\hline 25 & 223 & 121 \\
\hline 26 & 238 & 133 \\
\hline 27 & 213 & 126 \\
\hline 28 & 186 & 120 \\
\hline 29 & 187 & 118 \\
\hline 30 & 165 & 107 \\
\hline 31 & 140 & 84 \\
\hline 32 & 118 & 69 \\
\hline 33 & 97 & 59 \\
\hline 34 & 106 & 51 \\
\hline 35 & 72 & 43 \\
\hline 36 & 78 & 36 \\
\hline 37 & 63 & 29 \\
\hline 38 & 53 & 25 \\
\hline 39 & 43 & 20 \\
\hline 40 & 38 & 16 \\
\hline 41 & 27 & 11 \\
\hline 42 & 20 & 8 \\
\hline 43 & 13 & 5 \\
\hline 44 & 8 & 2 \\
\hline 45 & 5 & 1 \\
\hline 46 & 2 & 1 \\
\hline 47 & 1 & 0,2 \\
\hline 48 & 1 & 0,1 \\
\hline 49 & 0,3 & 0,3 \\
\hline Total & 81,1 & 47,9 \\
\hline Núm. de hijos por mujer & 2,87 & 1,62 \\
\hline
\end{tabular}


Figura 1. País Valenciano. Curvas de tasas de fecundidad general por edades, en 1975, 1979 y 1986.

1.000). Las diferencias de natalidad entre el norte y sur del País Valenciano eran más acentuadas en 1981: 13,8 por 1.000 en Castellón, 14,6 en Valencia y 16,2 en Alicante, es decir, como es norma generalizada, en el País Valenciano también se converge hacia el modelo común de tasas bajas.

Si comparamos las tasas de fecundidad entre 1975 y 1986 comprobamos un gran descenso de la fecundidad en las edades más fértiles, es decir entre 23 y 31 años de edad; también se ha producido una reducción de la maternidad tardía, de mujeres entre 35 y 49 años de edad, pues además de la disminución de tasas, sus nacimientos eran el 14\% del total en 1975 contra el 11\% en 1986. En el País Valenciano el número medio de hijos por mujer era de 2,87 en 1975, contra 1,62 en 1986, mientras que con posterioridad este índice ha continuado en descenso regular; a efectos comparativos, la tasa española era de 2,79 hijos por mujer en $1975,1,63$ en 1985 y 1,36 en $1990^{3}$.

Si la fecundidad valenciana es similar a la media española, en el uso de nuevos métodos o técnicas anticonceptivas el País Valenciano muestra una situación de control ligeramente menos efectiva que la media nacional; así se deduce de los datos ofrecidos por la Encuesta

3 Eurostat, Le capital humain européen à l'aube du 21e siècle. Actes de la Conference, Luxembourg, 1992, 605 pp. Cf. p. 526. 
de fecundidad de 1985 (INE, cuadro 2.3.1.1.), pues las mujeres sujetas a riesgo de embarazo que declaran usar métodos anticonceptivos se distribuyen del siguiente modo:

\begin{tabular}{|lcc|}
\hline Métodos utilizados & España(\%) & País Valenciano(\%) \\
\hline No usan ninguno & 52,5 & 44,2 \\
Píldora & 13,9 & 11,3 \\
DIU & 4,8 & 3,8 \\
Preservativo & 10,9 & 16,1 \\
Ritmo & 3,1 & 4,4 \\
Coitus interruptus & 13,0 & 18,1 \\
Otros métodos & 1,8 & 2,1 \\
\hline
\end{tabular}

\subsection{La mortalidad}

Las tasas de mortalidad actual reflejan las diferencias de las estructuras por edades: desde las tasas más bajas registradas en $1984-7,41$ por 1.000 en Alicante, 8,27 en Valencia y 9,42 en Castellón - se ha iniciado un inevitable ascenso a resultas del envejecimiento (en 1990 la tasa es 8,2 en Alicante, 9,0 en Valencia, y 9,8 en Castellón); no obstante, es preciso señalar que el País Valenciano mantiene en 1990 una tasa de mortalidad $(8,8)$ similar a la de España $(8,5)$, que se sitúa al nivel más bajo entre los países europeos - p.e. en Francia es de 9,3, en Reino Unido 11,2, en Suecia 11,1-, lo que se relaciona directamente con nuestra mayor juventud demográfica acumulada en los años de elevada natalidad.

En los años anteriores a 1986 la tasa general de mortalidad apenas varía en el País Valenciano - 9,2 por 1.000 en 1975, 8,4 en 1981, 8,5 en 1986-, sin embargo las tasas por edad y sexo sí experimentan cambios sensibles, especialmente entre los varones; en síntesis, entre 1979 y 1986 las variaciones más notables son: 1) Descenso de las tasas entre 0 y 16 años de edad, sobre todo en los varones, que en 1979 tenían tasas bastante más altas que las mujeres. 2) Entre 17 y 27 años de edad, las tasas femeninas no experimentan cambios significativos debido a sus bajos valores; los varones en cambio sufren un alza notable en su mortalidad, sin duda relacionada con los accidentes. 3) A partir de los 28 años de edad de nuevo se mejora en las tasas de mortalidad de ambos sexos, sobre todo entre los varones de 68 a 76 años de edad. En el País Valenciano se registra, en 1986, una normal sobremortalidad masculina - 9,0 por 1.000 en los varones, 8,0 en las mujeres-, equivalente a 108 varones por cada 100 mujeres (114 en 1979).

La mortalidad infantil del País Valenciano ha mejorado sensiblemente durante la última década: 16,3 por 1.000 nacidos en 1975, 13,3 en 1979 y 8,0 en 1986; la media de España es de 9,2 , cifra que coincide con la de los países europeos occidentales, aunque es superior a las tasas conseguidas en Europa del norte.

Según la mortalidad registrada en el País Valenciano en 1986, la edad de jubilación, es decir 65 años de edad, es alcanzada por el 78\% de los varones y el 89\% de las mujeres; más de la mitad de la población cumple los 76 años si son del sexo masculino y los 81 años si son del femenino.

Las causas de las defunciones en 1986, según el resumen de la clasificación internacional ofrecida por el INE, tienen una fuerte concentración en pocas catego- 
Figura 2. País Valenciano. Tasas de mortalidad (\%o) por sexos y edades, en 1979 y 1986.

rías: los óbitos atribuidos a enfermedades del aparato circulatorio suman en el País Valenciano el $46 \%$ del total; las producidas por tumores son el $21 \%$; las enfermedades del aparato respiratorio el $9 \%$ y las del digestivo el 6\%. Es decir, estos cuatro tipos de enfermedades representan más del $82 \%$ de las causas de mortalidad. También alcanza porcentaje notable el grupo «accidentes, envenenamientos y violencias» con el 5\% de las defunciones, de las cuales casi la mitad - 661 - son accidentes ocasionados por vehículos de motor.

Las principales causas de mortalidad señaladas para el País Valenciano tienen, por otra parte, un reparto desigual según sexos: las afecciones del aparato circulatorio producen el $52 \%$ de las muertes femeninas frente al $41 \%$ de las masculinas. Los tumores, en cambio, perjudican más a los varones, provocando el $24 \%$ de sus defunciones contra el $18 \%$ de las femeninas ó 143 varones por cada 100 mujeres; además, entre los varones esta causa de mortalidiad ha subido 4,3 puntos porcentuales entre 1979 y 1986, mientras entre las mujeres ha ganado 2,5 puntos. Igual ocurre con las muertes atribuidas a enfermedades respiratorias y digestivas, que suman el 9,8 y $6,6 \%$, repectivamente, de las muertes masculinas, y sólo el 7,7 y 4,8\% de los óbitos femeninos. En cuanto a los «accidentes, envenenamientos y violencias», los varones son las víctimas más frecuentes con 1.103 frente a 399 mujeres. 


\subsection{El excedente natural}

El excedente natural, de acuerdo con las evoluciones de la natalidad y mortalidad, se ha reducido en modo extremo: si en 1981 daba 23.949 nuevos habitantes en la región, en 1990 sólo alcanza a 5.667. Por provincias, al inicio de la década Valencia sumaba 12.528 habitantes de excedente natural, Alicante 9.570 y Castellón 1.851; la situación en 1990 es bien distinta: el mayor excedente corresponde ahora a Alicante con sólo 3.932 nuevos habitantes, seguida de Valencia con 2.406 y en Castellón es negativo, con -485; en esta provincia las defunciones son más numerosas que los nacimientos desde 1989, con fuerte crecimiento del balance negativo. Queda así patente que en Castellón — con la natalidad más baja y la mortalidad más alta de la región- el único factor de crecimiento demográfico, por el momento, se limita a la inmigración, ya que es posible que crezca el balance negativo del movimiento natural, sobre todo por aumento de la mortalidad. A efectos comparativos, según cifras provisionales de 1990, en España el crecimiento natural es de 1,68 por 1.000 , tasa que es superada por Alicante y 16 provincias más, todas ellas en la mitad sur de la península así como las insulares; Valencia — 1,13 por 1.000 — se sitúa por debajo de la media nacional, mientras la tasa de Castellón es negativa $(-1,08)$ junto con la de otras 21 provincias, todas en la mitad norte de la península, donde la situación más desfavorable es la que ofrece Galicia interior (Lugo -5,53 por 1.000), Zamora y Asturias.

\section{Cambios significativos en la distribución por edades}

Entre 1981 y 1991 los grupos de edad de la población valenciana muestran cambios de gran interés aplicado, con evoluciones divergentes en los grupos extremos (reducción de niños, aumento de ancianos) así como fuerte incremento de los adultos jóvenes; las modificaciones apuntadas conllevan evidentes repercusiones económicas tanto inmediatas como en el futuro próximo, y subrayan la insuficiencia de considerar sólo los efectivos totales al analizar la evolución demográfica.

Si el censo total del País Valenciano creció 210.363 habitantes de derecho $(5,8 \%)$ entre 1981 y 1991, el grupo infantil de 0-9 años de edad ha experimentado una fuerte disminución, pues en 1991 hay 185.450 niños menos de los que había en 1981 (vid. Cuadro III); esta disminución es resultado exclusivamente del descenso de nacimientos. En el extremo opuesto, el grupo de los que tienen 65 años o más ha aumentado durante la década en 111.320 habitantes — son el 13,5\% del censo total—, de los que casi la mitad (52.856) han engrosado el grupo de los «muy viejos» o personas con más de 75 años de edad, que totalizan 203.569 en 1991.

El tercer grupo con fuerte variación entre 1981 y 1991 es el de los adultos jóvenes, de 20 a 39 años de edad; su aumento ha sido de 176.193 habitantes. En una situación de pleno empleo el aumento de efectivos en este grupo de edades tendría repercusiones económicas muy positivas, pero actualmente tal incremento de adultos jóvenes sin duda afecta a las cifras de paro.

La estructura por edades de cada una de las tres provincias valencianas repite las tendencias señaladas para el conjunto regional, bien que con diferencias entre aquéllas. Así, Castellón presenta la estructura más envejecida, mientras en Alicante se encuentra la más joven. En efecto, el grupo infantil de 0-9 años tiene su menor importancia relativa en Castellón, con el 11\% de su censo en 1991, contra el 12,7\% en Alicante, aunque en Valencia es donde este grupo disminuyó más durante la década (vid. cuadro III). El grupo de los jubilados (65 años y más) en Alicante se eleva, en 1991, al 12,9\% de su censo total, 


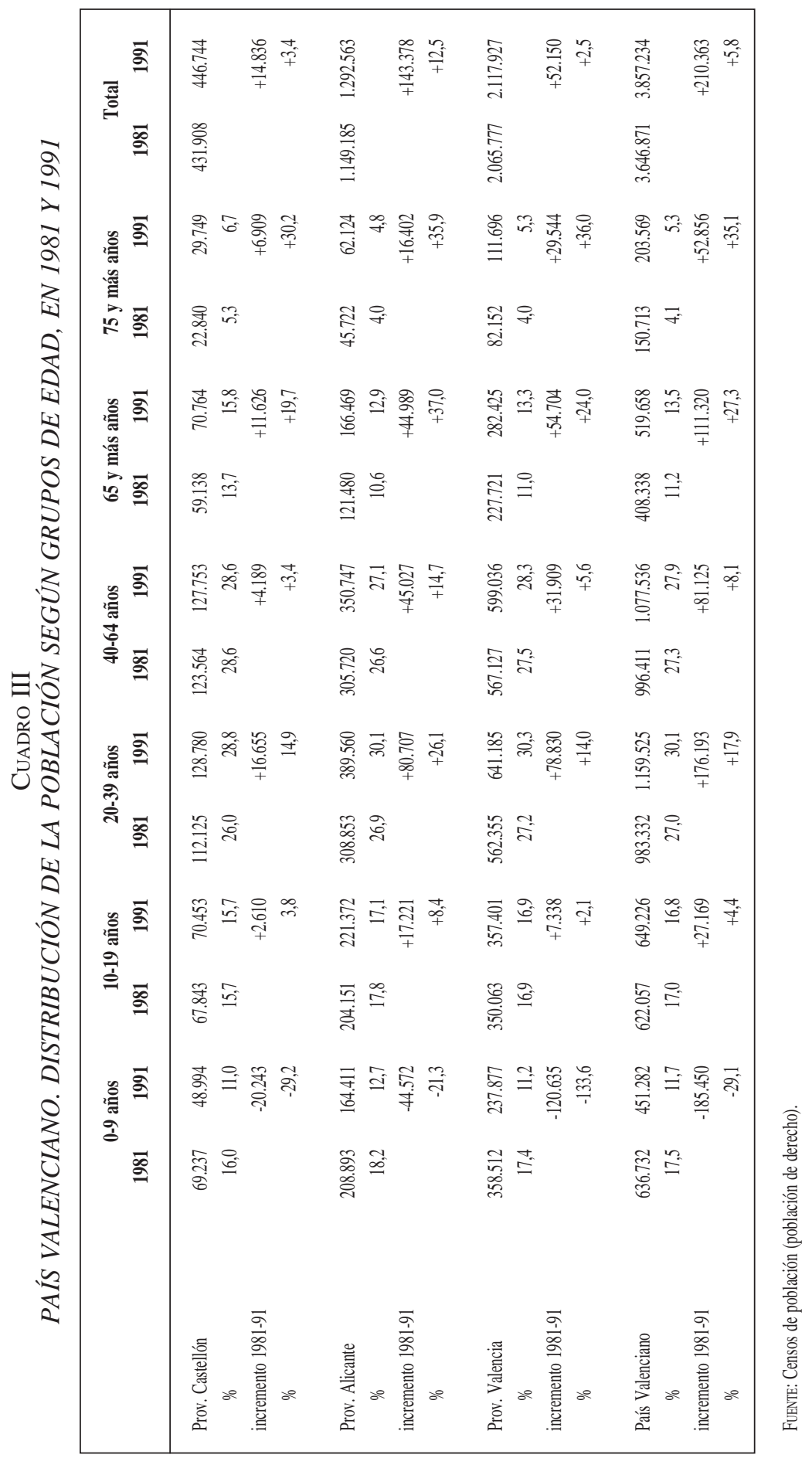


FigURA 3

mientras en Castellón son el 15,8\%, aunque las diferencias más significativas están en el grupo de los «muy viejos» (más de 75 años), pues en Castellón alcanzan el peso relativo más alto $(6,7 \%$ del censo) mientras los mayores incrementos relativos durante la década $(+36 \%)$ se producen en Valencia y Alicante.

En los dos grupos de adultos (20-64 años) destacan los mayores incrementos absolutos registrados en la provincia de Alicante $(+125.734$ habitantes entre 1981 y 1991), superiores incluso a los de Valencia (+110.739), pese a que la demarcación meridional sólo participa con un tercio del censo regional contra casi el 55\% de Valencia (población de derecho): las cifras más altas del incremento de adultos en Alicante son buen reflejo de su mayor inmigración durante la década, así como de su alta natalidad anterior; obviamente los mayores incrementos de adultos también se traducen en las cifras de parados de la provincia de Alicante, ya que en 1990 éstos superan el $42 \%$ del total registrado en la Comunidad ${ }^{4}$.

4 Institut Valencià d'Estadística, Anuari Estadístic Comunitat Valenciana, 1990. 


\section{Las variaciones demográficas en los municipios valencianos}

\subsection{La concentración demográfica}

Durante el último intercensal la inmigración neta ha sido relativamente positiva en las tres provincias valencians, con una suma de saldos de +111.616 habitantes en 1986-90, frente a los +34.429 de 1981-85. Este incremento reciente de las migraciones extraprovinciales también afecta de modo notable a escala municipal, tal como se deduce de las variaciones censales municipales, registradas entre 1981-86 y 1986-91. En concreto, esta reactivación de los movimientos migratorios se traduce en una mayor concentración demográfica en las áreas de aumento censal y también en una mayor velocidad de la despoblación en los municipios con disminución censal. En efecto los municipios con aumento censal en 1981-86 eran 269, frente a 207 en 1986-91, mientras la variación del censo regional pasó de $+3,4 \%$ a $+4,0 \%$; por el contrario, durante el primer período disminuyeron su censo 264 municipios, frente a 329 en el segundo.

La misma tendencia de concentración demográfica se observa en cada una de las circunscripciones provinciales, bien que con evidentes particularidades (cuadro IV): los municipios con aumento censal disminuyen su número en la segunda mitad de los años 1980 cuando su crecimiento demográfico es inferior a $2 \%$ anual, mientras que aumenta el número de los que registran máximo crecimiento (más de $3 \%$ anual), sobre todo en Alicante.

Por el contrario, los municipios con disminución censal se incrementan durante el segundo quinquenio en todos los umbrales, con la particularidad de que el aumento es

CUADRO IV

PAÍS VALENCIANO. NÚMERO DE MUNICIPIOS CON AUMENTO CENSAL (\% ANUAL)

\begin{tabular}{|c|c|c|c|c|c|c|c|c|}
\hline \multirow[b]{2}{*}{ Provincias } & \multicolumn{2}{|c|}{0,1 a 0,9} & \multicolumn{2}{|c|}{1,0 a 1,9} & \multicolumn{2}{|c|}{2,0 a 2,9} & \multicolumn{2}{|c|}{ 3,0 y más } \\
\hline & $1981-86$ & $1986-91$ & $1981-86$ & $1986-91$ & $1981-86$ & $1986-91$ & $1981-86$ & $1986-91$ \\
\hline Alicante & 41 & 34 & 30 & 21 & 8 & 9 & 9 & 15 \\
\hline Castellón & 33 & 15 & 2 & 2 & 4 & 5 & 4 & 3 \\
\hline Valencia & 89 & 72 & 37 & 19 & 6 & 4 & 6 & 8 \\
\hline Total & 163 & 121 & 69 & 42 & 18 & 18 & 19 & 26 \\
\hline Incremento, $\%$ & & $-25,8$ & & $-39,1$ & & 0,0 & & $+36,8$ \\
\hline \multicolumn{9}{|c|}{ NÚMERO DE MUNICIPIOS CON DISMINUCIÓN CENSAL (\% ANUAL) } \\
\hline & \multicolumn{2}{|c|}{0,1 a 0,9} & \multicolumn{2}{|c|}{1,0 a 1,9} & \multicolumn{2}{|c|}{2,0 a 2,9} & \multicolumn{2}{|c|}{ 3,0 y más } \\
\hline Provincias & $1981-86$ & $1986-91$ & $1981-86$ & $1986-91$ & $1981-86$ & $1986-91$ & $1981-86$ & $1986-91$ \\
\hline Alicante & 17 & 24 & 18 & 19 & 10 & 10 & 5 & 6 \\
\hline Castellón & 34 & 31 & 27 & 31 & 15 & 18 & 16 & 30 \\
\hline Valencia & 75 & 89 & 28 & 37 & 10 & 16 & 9 & 18 \\
\hline Total & 126 & 144 & 73 & 87 & 35 & 44 & 30 & 54 \\
\hline Incremento, \% & & $+14,3$ & & $+19,2$ & & $+25,7$ & & $+80,0$ \\
\hline
\end{tabular}


mayor cuanto más intensa es la despoblación: así, los municipios que disminuyen su censo menos del $1 \%$ anual crecen el $14 \%$ entre 1981-86 y 1986-91, mientras que los que sufren pérdidas superiores al 3\% anual se incrementan en un 80\%, especialmente en Castellón donde se concentran más de la mitad de todos los municipios con despoblación máxima.

La concentración demográfica se produce a favor de los municipios con censo superior a 10.000 habitantes (en 1991 son 74, de un total de 539), pues si en 1981 su población representaba el 75,9\% del total, en 1991 alcanzan el 77,9\%, bien que parte de este aumento se debe a los 3 nuevos municipios urbanos; si contabilizamos por separado la «población urbana», su crecimiento intercensal es del 9,1\% (+275.386 habitantes de hecho), mientras el aumento del conjunto de la población regional es el 7,6\% (+277.076 habitantes).

Durante el intercensal 1981-86 las variaciones censales municipales con valores entre $+2 \%$ y $-1 \%$ anual son las que predominan y cubren - entremezcladas - un gran espacio que se extiende entre Oropesa y la frontera murciana, pero que excluyen las comarcas del interior de Valencia (Los Serranos, la Plana de Utiel-Requena y el Valle de Ayora) así como los municipios de la montaña alicantina. En las últimas comarcas indicadas, así como en casi toda la provincia de Castellón exterior a La Plana, están generalizados los municipios en retroceso demográfico. En cualquier caso, son pocos y con escasa población los municipios que alcanzan variaciones extremas, tanto positivas como negativas ${ }^{5}$.

Durante el intercensal 1986-91 el mapa municipal de crecimiento demográfico se ha «enfriado» muy notablemente, en lo que influye, por un lado, el fortísimo y generalizado retroceso del crecimiento natural, y, por otro, la concentración del crecimiento demográfico en un menor número de municipios.

Las variaciones positivas entre 1,0 y 1,9\% anual que en 1981-86 alcanzaban amplia difusión en municipios no litorales del centro y sur de la región, en 1986-91 han experimentado fortísimo retroceso, sobre todo en Valencia, sustituidos por crecimientos mínimos o negativos. Sobre el conjunto regional destaca la multiplicación de los municipios con retroceso demográfico en 1986-91: las comarcas interiores de Castellón y Valencia, así como la montaña alicantina, han intensificado mucho su despoblación, tanto por agravamiento de las tasas negativas como por la ampliación de las «manchas» que forman este tipo de municipios; es llamativo el «avance» de los municipios con variaciones censales negativas, pues ahora también se generalizan en las áreas no montañosas como la Vall d'Albaida, la Ribera del Xúquer, el Camp de Túria o el Camp de Morvedre. No obstante, es en casi toda la provincia de Castellón donde las variaciones censales negativas se han agravado más, singularmente en el Alto Mijares, Alto Palancia, els Ports y l'Alt Maestrat; entre 1986 y 1991 en Castellón han aumentado su censo 25 municipios, mientras han sido 110 los que lo han disminuido y de éstos 30 con tasas anuales entre -3 y $-6,5 \%$.

Durante el último intercensal los incrementos mayores se encuentran fundamentalmente en los municipios turísticos del litoral alicantino, a los que se añaden los periurbanos de la ciudad de Alicante. El área metropolitana de Valencia se ha revitalizado significativamente (municipios industriales y residenciales) respecto al quinquenio anterior, e igual ha sucedido con la ciudad de Castellón y su entorno norte inmediato.

Las cuatro ciudades más pobladas del País Valenciano en 1986-91 han revitalizado sensiblemente su crecimiento censal respecto al quinquenio anterior; la excepción es Alicante donde ha continuado el retroceso de la tasa de aumento: si en 1960-70 alcanzó $5,2 \%$ anual medio, en 1986-91 lo ha reducido a 0,7\%, aunque «en compensación» los

5 El mapa de variaciones censales municipales en 1981-86, fue publicado en Gozálvez Pérez, V., «La población valenciana en 1986: la evidencia de una fuerte y abierta crisis», Cuadernos de Geografía, núm. 42, Valencia, 1987, pp. 149-158. 
Figura 4. Variación censal anual entre 1986 y 1991, en los municipios del País Valenciano.

municipios de su área metropolitana han experimentado un fortísimo aumento relativo en el último quinquenio: Sant Vicent ha crecido 3,5\% anual, Mutxamel 4,6\%, Sant Joan 4,0\% y el Campello 3,3\%. Elche continua registrando el mayor aumento censal (1,6\% anual en 1986-91) entre los municipios más poblados, debido a que mantiene un crecimiento natural relativamente muy saneado (5,6 por 1.000 en 1986-90 ó +6.378 habitantes), fruto de su juventud demográfica, es decir se suman una natalidad relativamente alta (11,6 por 1.000 
en 1990) a escala regional y, sobre todo, una mortalidad muy baja (6,0 por 1.000); además, su inmigración neta parece haberse revitalizado mucho (+7.826 habitantes en 1986-90, frente a +211 en el quinquenio anterior).

Las mejoras en el crecimiento demográfico de las capitales Castellón ( $0,5 \%$ anual en $1981-86,1,3 \%$ en 1986-91) y Valencia $(-0,3$ y $+1,1$, respectivamente), parecen sólidas, pues van acompañadas de parte de las de municipios de sus entornos inmediatos. Además, en la capital regional ${ }^{6}$ la variación censal de 1986-91 ha mejorado en casi todos sus distritos censales respecto a 1981-86: así, las variaciones negativas del primer período (en 13 distritos) se han aminorado (en 5 distritos) o han cambiado a positivas (en 7 distritos), mientras que de los 6 distritos que en 1981-86 tuvieron variación positiva, 5 la mantienen, y de estos 4 aumentan mucho su crecimiento. Todo lo indicado parece confirmar la solidez del crecimiento demográfico de la capital regional, pues afecta a casi todo el plano de la ciudad así como a la mayor parte de su entorno metropolitano.

Las tres capitales y Elche en 1986-91 suman un aumento de 71.300 habitantes, de los que más de la mitad en la ciudad de Valencia y una quinta parte en Elche; de los cuatro, Alicante es el único municipio que crece menos que la media regional $(+4,0 \%)$, mientras que Elche es el único que dobla $(8,2 \%)$ dicha media.

\subsection{Convergencia en tasas de natalidad bajas}

Los mapas municipales de natalidad, mortalidad y crecimiento vegetativo de 1985-87 se han obtenido con las medias del trienio y la población de 1986. El mapa de la natalidad municipal en el período $1975-79^{7}$ se caracterizaba por fortísimos contrastes entre las envejecidas zonas rurales interiores o montañosas, víctimas de un antiguo y continuado éxodo rural, con gran abundancia de tasas de natalidad inferiores a 10 por 1.000 , y las zonas de alto crecimiento poblacional, inmigratorias, donde la natalidad oscilaba entre 17 y 23 por 1.000 . El mapa de 1985-87, muestra un cambio drástico en la natalidad municipal, al desaparecer las tasas superiores al 15 por 1.000, que eran las protagonistas de los violentos contrastes observados en 1975-79 entre el norte y el sur, y entre las áreas litorales e interiores de la región.

Así, los municipios con natalidad inferior al 10 por 1.000 eran 178 en 1975-79 contra 291 en 1985-87 — la natalidad media regional en el último período es de 11,4 por 1.000, de los que 102 pertenecen a la provincia de Castellón (Cuadro V). Estos municipios con natalidad más baja, en 1975-79 formaban manchas más o menos discontinuas en las comarcas montañosas e interiores, mientras en 1985-87 abarcan extensas áreas compactas, que se han ampliado desde las ya existentes cinco años atrás, e incluso alcanzan a buen número de los municipios turístico-litorales del norte de Alicante y a bastantes de los que forman el área metropolitana de Valencia. Estos municipios con natalidad inferior a 10 por 1.000 en 1985-87 incluyen al 44\% de los de Alicante, al 49\% de los de Valencia y al 76\% de los de Castellón.

Los municipios con natalidad entre 10 y 14,9 por 1.000 en $1985-87$ totalizan el $43 \%$ de los de la región, e incluyen a casi todos los que tienen tasa superior a 10\%o; se encuentran

6 Las cifras de los distritos censales de Valencia proceden del Anuari Estadístic de la Ciutat de València. 1991, que utiliza población de derecho, mientras en el resto de las variaciones censales ofrecidas en este estudio se ha utilizado la población de hecho. En conjunto el municipio de Valencia en 1991 tiene 752.909 habitantes de derecho y 777.427 habitantes de hecho.

7 GOZÁLVEZ PÉREZ, V.: «Cambios recientes en la población valenciana», ap. Estudis sobre la població del País Valencià, vol. II, Valencia, Institució Valenciana d'Estudis i Investigació, 1988, pp. 671-706. 
FiguRA 5. Natalidad media del período 1985-87, en los municipios del País Valenciano.

en este umbral parte de los municipios interiores con demografía menos dinámica y casi todos los que han experimentado durante los quinquenios precedentes los mayores crecimientos censales, es decir la Plana de Castelló, casi todos los de la franja litoral de Valencia, y en Alicante las comarcas industriales, el Bajo Segura y l'Alacantí.

Con natalidad de 15\%o o más había 213 municipios en 1975-79, pero en 1985-87 sólo quedan 16, con una distribución espacial muy dispersa, es decir, no forman áreas de natalidad más elevada, sino que son «restos» de tendencias pasadas en vías de desapari- 


\section{CuADRo V}

DISTRIBUCIÓN DE LOS MUNICIPIOS DEL PAÍS VALENCIANO SEGÚN SUS TASAS DE NATALIDAD EN 1985-87 (\%o)

\begin{tabular}{|lrrrrr|}
\hline Natalidad & $\begin{array}{c}\text { Provincia } \\
\text { Alicante }\end{array}$ & $\begin{array}{c}\text { Provincia } \\
\text { Valencia }\end{array}$ & $\begin{array}{c}\text { Provincia } \\
\text { Castellón }\end{array}$ & \multicolumn{2}{c|}{ País Valenciano } \\
\hline$<10$ & 61 & 128 & 102 & 291 & 54,4 \\
$10-14,9$ & 70 & 128 & 30 & 228 & 42,6 \\
$15-17$ & 6 & 5 & 2 & 13 & 2,4 \\
$17,1-19,9$ & 1 & 1 & 0 & 2 & 0,4 \\
20 y más & 0 & 1 & 0 & 1 & 0,2 \\
\hline Total & 138 & 263 & 134 & 535 & 100,0 \\
\hline
\end{tabular}

ción.

En las cuatro grandes ciudades de la región la natalidad de 1985-87 es de 14,9 por 1.000 en Elche, $12 \%$ en Alicante, 11,8 en Castellón y 10,8 en Valencia; en relación a 1975-79 Elche ha perdido 7,7 puntos. Es decir, mientras en la segunda mitad de los años 1970 la natalidad de estas ciudades estaba influenciada al alza por las características sociodemográficas de sus masivas y recientes inmigraciones, en 1985-87 esta influencia positiva se ha reducido sensiblemente o es nula, en parte por haber desaparecido la inmigración laboral procedente de las regiones más natalistas y por la convergencia hacia una natalidad homogénea en tasas bajas.

La homogeneidad actual de la natalidad queda muy patente al comparar las tasas según el contingente demográfico de los municipios, tal como se indica en el cuadro que sigue:

PAÍS VALENCIANO. NATALIDAD (\%o) EN LOS DISTINTOS GRUPOS DE MUNICIPIOS, EN 1975 Y 1986

\begin{tabular}{|lccc|}
\hline $\begin{array}{l}\text { Habitantes } \\
\text { del municipio }\end{array}$ & $\mathbf{1 9 7 5}$ & Natalidad & Variación, \% \\
\hline 100.000 & 20,4 & $\mathbf{1 9 8 6}$ & -42 \\
$40.000-100.000$ & 21,5 & 11,8 & -43 \\
$20.000-40.000$ & 20,0 & 12,2 & -40 \\
$10.000-20.000$ & 19,4 & 12,0 & -40 \\
$5.000-10.000$ & 17,7 & 11,6 & -36 \\
$2.000-5.000$ & 16,0 & 11,4 & -37 \\
$<2.000$ & 12,6 & 10,0 & -29 \\
Región & 19,3 & 8,9 & -40 \\
\hline
\end{tabular}

Fuente: INE, Movimiento natural de la población de España; Padrones de población.

Tanto en 1975 como en 1986 todos los grupos de municipios «urbanos» tienen natalidad superior a la media regional; por el contrario, los de censo inferior a 10.000 habitantes registran natalidad más baja que la media del País, con las menores tasas en los municipios 
rurales. Es clara pues, la influencia de las migraciones en la natalidad, a través de las costumbres arrastradas desde el lugar de origen y de la modificación de la estructura de edades. Disminuidos los éxodos rurales y generalizada la tendencia a limitar el número de hijos, en 1986 las tasas se «uniformizan» en las cifras más bajas en los distintos tipos de poblamiento: si en 1975 había 8,9 puntos entre las tasas extremas, en 1986 la diferencia es sólo de 3,3 puntos.

\subsection{Distribución geográfica de la mortalidad}

Las diferencias municipales de la mortalidad en 1985-87 responden a las estructuras por edades de la población, lo que a su vez es consecuencia de los movimientos migratorios, tanto de signo positivo como negativo.

En contra de lo que ocurre con los mapas de natalidad de 1975-79 y 1985-87, que registran descensos muy acentuados, los mapas de mortalidad confeccionados para las mismas fechas muestran diferencias muy tenues, que están referidas especialmente al aumento del número de municipios con tasas inferiores al 8 por 1.000 (82 en la primera

\section{Cuadro VII}

PAÍS VALENCIANO. DISTRIBUCIÓN DE LOS MUNICIPIOS SEGÚN SUS TASAS DE MORTALIDAD (\%o) EN 1985-87

\begin{tabular}{|lcccc|}
\hline Mortalidad & $\begin{array}{c}\text { Provincia } \\
\text { Alicante }\end{array}$ & $\begin{array}{c}\text { Provincia } \\
\text { Castellón }\end{array}$ & $\begin{array}{c}\text { Provincia } \\
\text { Valencia }\end{array}$ & $\begin{array}{c}\text { País } \\
\text { Valenciano }\end{array}$ \\
\hline$<8$ & 49 & 8 & 60 & 117 \\
$8-10,9$ & 34 & 21 & 94 & 149 \\
$11-13,9$ & 21 & 35 & 71 & 127 \\
$14-17$ & 13 & 27 & 22 & 62 \\
$>17$ & 21 & 43 & 16 & 80 \\
\hline Total & 138 & 134 & 263 & 535 \\
\hline
\end{tabular}

fecha contra 117 en la segunda).

Las áreas inmigratorias, con acentuado rejuvenecimiento de su población, son las de mortalidad más baja, generalmente entre 7 y 8 por 1.000 , e incluso con tasas menores sobre todo en el área metropolitana de Valencia y comarcas industriales de Alicante; también en el litoral turístico y Bajo Segura se registran tasas muy bajas, en el primer caso debidas a la inmigración y en el segundo a su alta natalidad.

En el extremo opuesto se sitúan los municipios con mortalidad superior a 17 por 1.000. Aunque su volumen demográfico es escaso, estos municipios sí cubren extensas áreas del País, donde se acumulan todos los índices demográficos negativos (baja natalidad, despoblamiento, envejecimiento, etc.). Destaca la peor situación de la provincia de Castellón, pues de 117 municipios que en la región tienen mortalidad inferior a 8\%o, sólo 8 pertenecen a Castellón, mientras de los 80 municipios con tasas superiores a 17, Castellón suma 43. La provincia de Alicante es la que presenta áreas más contrastadas entre alta mortalidad —en los pequeños municipios de la montaña — y baja mortalidad — resto de la provincia—. Las tasas de mortalidad intermedias, entre 8 y 13,9 por 1.000 son las más repetidas en todo el País, aunque en el norte también son muy numerosos los municipios con mortalidad entre 
Figura 6. Mortalidad media del período 1985-87, en los municipios del País Valenciano.

14 y 17 por 1.000 .

En resumen, la distribución municipal de la mortalidad presenta actualmente fuertes contrastes. Las tasas bajas prodominan en las áreas dominadas por la población urbana, tanto como resultado del tamaño de los núcleos como por sus actividades secundarias y 
terciarias; las tasas son más bajas cuanto más reciente y voluminosa es la inmigración, pues esta ha provocado el rejuvenecimiento de la estructura demográfica. Al contrario, en las áreas agrícolas y rurales, con núcleos pequeños, las tasas de mortalidad son altas o muy altas, según la antigüedad del éxodo rural que hayan experimentado, ya que este ha deteriorado la estructura por edad y sexo.

FiguRA 7. Crecimiento vegetativo medio del período 1985-87, en los municipios del País Valenciano. 
El crecimiento natural del País Valenciano alcanzó tasas superiores al 1,1\% anual entre 1957 y 1976, período el más uniformemente saneado del siglo XX; después de 1977 el continuado descenso de la natalidad ha sido el responsable de la reducción del saldo natural hasta el $0,3 \%$ de 1986 , tendencia negativa que ha continuado posteriormente $(0,15 \%$ en 1990$)$.

En 1985-87 el País Valenciano contabiliza 237 municipios con excedente natural positivo y 298 con tasas negativas. Los municipios con tasas superiores al 10 por 1.000 prácticamente han desaparecido, sólo 7 frente a 74 en 1975-79; estas tasas más altas en parte han sido sustituidas por las de valores entre 5 y 10 por 1.000 (Valle del Vinalopó, Foia de Castalla, Bajo Segura y parte del oeste del área metropolitana de Valencia), y sobre todo por las tasas positivas inferiores a 5\%o. Estas últimas son, en 1985-87, las tasas más ampliamente representadas, y afectan a casi todos los municipios del litoral turístico, así como a la mayoría de los de economía industrial y agraria más pujantes de Castellón y Valencia, y también a una amplia representación de municipios interiores del centro y sur

CuAdro VIII

PAÍS VALENCIANO. DISTRIBUCIÓN DE LOS MUNICIPIOS SEGÚN SUS TASAS DE EXCEDENTE NATURAL EN 1985-87 (\%०)

\begin{tabular}{|lccccc|}
\hline $\begin{array}{l}\text { Excedente } \\
\text { Positivo }\end{array}$ & $\begin{array}{c}\text { Provincia } \\
\text { Alicante }\end{array}$ & $\begin{array}{c}\text { Provincia } \\
\text { Castellón }\end{array}$ & $\begin{array}{c}\text { Provincia } \\
\text { Valencia }\end{array}$ & $\begin{array}{c}\text { País } \\
\text { Valenciano }\end{array}$ & $\%$ \\
\hline$>10$ & 4 & 1 & 2 & 7 & 1 \\
$5-10$ & 37 & 3 & 23 & 63 & 12 \\
$<5$ & 32 & 23 & 112 & 167 & 31 \\
Total & 73 & 27 & 147 & 237 & 44 \\
Excedente & & & & & \\
Negativo & 27 & 24 & 81 & 132 & 25 \\
$<5$ & 24 & 32 & 31 & 87 & 16 \\
$5-10$ & 14 & 51 & 14 & 79 & 15 \\
$>10$ & 65 & 107 & 126 & 298 & 56 \\
\hline Total & & & & & \\
\hline
\end{tabular}

del País; suman casi un tercio de los municipios valencianos.

Los municipios con excedente natural negativo han aumentado de 232 en 1975-79 a 298 en 1985-87, y su incremento es tanto mayor cuanto más negativa es la tasa. Como ocurre con otros índices que indican degradación demográfica, los municipios con saldo natural negativo se han expandido en manchas de aceite a partir de las áreas ya afectadas por esas tasas en 1975-79, al mismo tiempo que también amplían las áreas con las tasas más negativas.

La degradación de la dinámica demográfica que se deriva de procesos emigratorios antiguos e intensos queda bien reflejada en el mapa municipal de crecimiento natural negativo, cuyos valores más extremos se concentran en la montaña alicantina, en las 
comarcas interiores del centro y, sobre todo, en la provincia de Castellón, donde las tasas negativas afectan al $80 \%$ de los municipios (contra el $47 \%$ de los de Alicante y el $48 \%$ de los de Valencia), es decir todas sus comarcas con la excepción de la Plana y de los municipios estrictamente litorales de la mitad norte; el Alto Mijares y en parte el Alto Palancia tienen la mayor concentración regional de municipios con crecimiento vegetativo muy negativo, o sea, inferior a $-10 \%$; de los 79 de todo el País, 51 pertenecen a la provincia septentrional. Como se observa, la situación más degradada se extiende a casi toda la provincia de Castellón no litoral, al Rincón de Ademuz y parte de Los Serranos, así como a la montaña de Alicante; la esperanza de revitalización demográfica es aquí, por el momento, obviamente muy escasa.

$* * * * * *$

En resumen, la década de los años 1980 ofrece un balance relativamente positivo para el censo demográfico valenciano, si el punto de referencia es la evolución experimentada por las distintas regiones españolas. Sin embargo, tal conclusión debe aminorar mucho su optimismo al analizar la evolución de las distintas variables geodemográficas. Así a lo largo de la década - y año a año— se ha reducido drásticamente el excedente natural, casi exclusivamente por disminución de los nacimientos, hasta convertirse en negativo en la provincia de Castellón y en el 56\% de los municipios del País; en consecuencia, aunque la región creció en 277.076 habitantes entre 1981 y 1991, el grupo infantil de 0-9 años de edad disminuyó en 185.450 niños, mientras el grupo con 65 años y más aumentó en 111.320 habitantes, todo lo cual provoca un notable aumento del envejecimiento (en 1991 el grupo con 65 años o más representa el 13,5\% del censo valenciano).

La inmigración neta se ha reactivado considerablemente en el último quinquenio, aunque sería preciso estudiar las estructuras por edades y socioprofesional de los inmigrantes para evaluar su incidencia económica y demográfica. Durante el último quinquenio se han iniciado dos nuevas tendencias: aumento de la tasa de mortalidad debido al envejecimiento, lo que unido a la baja natalidad hace peligrar la existencia de excedente natural positivo; la provincia de Alicante, aunque es la que continúa teniendo la mayor tasa de crecimiento demográfico, ha perdido atracción inmigratoria tanto en cifras absolutas como en relativas sobre el resto de la región. Esta disminución de la atracción inmigratoria de Alicante que evidencia las dificultades de su economía, junto con un gran aumento de su población adulta-joven, llevan a esta circunscripción a unas tasas de paro mayores que en el centro y norte de la región (19,5\% en Alicante, 12,3\% en Castellón, 14,4 \% en Valencia, 17,0 en España, $4^{\circ}$ trimestre 1991, según EPA).

Finalmente hay que señalar que durante el último quinquenio 1986-91 en el País Valenciano se ha reactivado la despoblación de sus municipios con localizaciones y estructuras demográficas más desfavorables, mientras aumenta la concentración de la población en los municipios urbanos. 